\title{
Qualité Microbiologique, Propriétés Physicochimiques et Profil Sensoriel de Miels de la Région du Worodougou, Côte d'Ivoire
}

\section{Coulibaly Bakary,}

Maître-Assistant, Enseignant-Chercheur, Laboratoire d'Agrovalorisation, Département de Biochimie-Microbiologie, UFR Agroforesterie,

Université Jean Lorougnon Guédé, Daloa, Côte d'Ivoire

Diomandé Massé,

Konaté Ibrahim,

Maître de Conférences, Enseignant-Chercheur, Laboratoire

d'Agrovalorisation, Département de Biochimie-Microbiologie, UFR

Agroforesterie, Université Jean Lorougnon Guédé, Daloa, Côte d'Ivoire

\section{Bohoua Louis Guichard,}

Professeur Titulaire, UFR Sciences et Technologie des Aliments,

Université Nangui Abrogoua, Abidjan, Côte d'Ivoire

\section{Resume}

Le présent travail a été réalisé dans la région du Worodogou (Côte d'Ivoire). L'objectif de l'étude était d'évaluer la qualité microbiologique et les caractéristiques physico-chimiques et sensorielles de ces miels. La méthode a consisté à rechercher et dénombrer les microorganismes capables d'altérer la qualité organoleptique et sanitaire de ces miels sur des milieux de culture. L'analyse des paramètres physico chimiques concernait la détermination de la densité des miels, le pH, l'acidité, le taux de sucre, le taux de matière sèche et l'analyse sensorielle. Les résultats montrent un taux de flore mésophile aérobie totale compris entre $1 \mathrm{ufc} / \mathrm{g}$ et $66,14 \mathrm{ufc} / \mathrm{g}$. Les miels analysés ne relèvent pas de contamination par les coliformes. Tous les miels sont dépourvus de levures et moisissures excepté le miel du commerce avec un taux de contamination de 2 ufc / g. On note une contamination par les Staphylocoques du miel provenant de WONGUE (MW) $(3,64$ à 4,63 ufc/g). Aucune salmonelle n'a été révélée dans l'ensemble des miels. L'analyse physico-chimique a montré des densités de miels variant de $1,06 \pm 0,069$ à $1,22 \pm 0,089 \mathrm{~g} / \mathrm{cm}^{3}$. Les $\mathrm{pH}$ des échantillons varient entre 3,7 $\pm 0,1$ et 4,77 \pm 0,06 . Le miel de BOBI a la plus faible teneur en glucose $(41,71 \pm 6,54 \%)$ comparée à celle du miel du commerce, de FORONA et de WONGUE (45,50 
$\pm 0.0 \%$ ). Les valeurs de l'acidité totale des miels oscillent entre $16,67 \pm 2,89$ et $33,33 \pm 2,89 \mathrm{meq} / \mathrm{kg}$. Les extraits secs réfractométriques (ERS) ont varié de $21 \pm 0,44$ à $24 \pm 0,1 \%$. Les couleurs des miels sont le jaune (MC), le marron (MB) et le marron foncée (MF, MW). La saveur des miels est la saveur sucrée. Le gout des miels se situe entre très désagréable à extrêmement agréable Comparés aux normes internationales, on peut affirmer que les miels de la région du Worodougou (Côte d'Ivoire) sont de qualités acceptables.

Mots clés: Miel, Qualité microbiologique, Analyse physicochimique, Couleur, Saveur, Côte d'Ivoire

\title{
Microbiological Quality, Physicochemical Properties and Sensory Profile of Honeys from the Worodougou Region, Côte d'Ivoire
}

\section{Coulibaly Bakary,}

Maître-Assistant, Enseignant-Chercheur, Laboratoire d'Agrovalorisation, Département de Biochimie-Microbiologie, UFR Agroforesterie,

Université Jean Lorougnon Guédé, Daloa, Côte d'Ivoire

\section{Diomandé Massé, Konaté Ibrahim,}

Maître de Conférences, Enseignant-Chercheur, Laboratoire d'Agrovalorisation, Département de Biochimie-Microbiologie, UFR Agroforesterie, Université Jean Lorougnon Guédé, Daloa, Côte d'Ivoire

\section{Bohoua Louis Guichard,}

Professeur Titulaire, UFR Sciences et Technologie des Aliments, Université Nangui Abrogoua, Abidjan, Côte d'Ivoire

\begin{abstract}
This work was done in the Worodogou region (Ivory Coast). The objective of the study was to evaluate the microbiological quality and the physicochemical and sensory characteristics of these honeys.The method consisted of looking for and counting microorganisms capable of altering the organoleptic and health quality of these honeys on culture media. The analysis of physico-chemical parameters concerned the determination of honey density, $\mathrm{pH}$, acidity, sugar content, dry matter content and sensory analysis. The results show a total aerobic mesophilic flora content of between $1 \mathrm{cfu} / \mathrm{g}$ and $66.14 \mathrm{cfu} / \mathrm{g}$. The honeys analyzed do not include coliform contamination.
\end{abstract}


All honeys are free of yeasts and molds except for commercial honey with a contamination rate of $2 \mathrm{cfu} / \mathrm{g}$. Honey Staphylococci contamination was reported from WONGUE (MW) (3.64 to $4.63 \mathrm{CFU} / \mathrm{g}$ ). No salmonella was found in all honeys. Physicochemical analysis showed honey densities ranging from $1.06 \pm 0.069$ to $1.22 \pm 0.089 \mathrm{~g} / \mathrm{cm} 3$. The $\mathrm{pH}$ of the samples varies between $3.7 \pm 0.1$ and $4.77 \pm 0.06$. BOBI honey has the lowest glucose content $(41.71 \pm 6.54 \%)$ compared to commercial honey, FORONA and WONGUE $(45.50 \pm 0.0 \%)$. The total acidity values of honeys ranged from $16.67 \pm 2.89$ to $33.33 \pm 2.89 \mathrm{meq} / \mathrm{kg}$. Refractometric dry extracts (RHS) ranged from 21 \pm 0.44 to $24 \pm 0.1 \%$. The colors of the honeys are yellow (MC), brown (MB) and dark brown (MF, MW). The flavor of honey is the sweet taste. The taste of honeys is between very unpleasant to extremely pleasant Compared to international standards, it can be said that honeys from the region of Worodougou (Ivory Coast) are acceptable qualities.

Keywords: Honey, Microbiological quality, Physicochemical analysis, Color, Flavor, Ivory Coast

\section{Introduction}

Dès la préhistoire, le miel produit par les abeilles a été récolté par l'homme de façon très artisanale pour ses propriétés nutritives et thérapeutiques. Aujourd'hui, l'apiculteur s'efforce d'obtenir de ses abeilles un produit de qualité en quantité suffisante pour répondre à la demande des consommateurs, et ce malgré la tendance à l'affaiblissement et à la mortalité des colonies d'abeilles dans le monde entier. Actuellement, le miel est perçu par le grand public comme un aliment naturel, non pollué et bénéfique pour la santé (Lequet, 2010). Pratiquement tous les pays du monde en font d'une variété ou d'une autre, mais ces pays ne sont pas tous des grands acteurs sur le marché international. Les plus grands pays producteurs de miel sont la Chine, les Etats Unis et l'Argentine. Certains pays d'Afrique de l'Est sont aussi de grands acteurs sur le marché international, mais le continent africain en général est peu représenté à l'échelle mondiale. Le marché international du miel total environ 1.6 million de tonnes en 2011, et ce chiffre serait en hausse, selon les données de la FAO, due à l'augmentation de la production en Chine ( $+60 \%$ en 10ans) et de l'Union Européenne ( $+30 \%$ en 10 ans). On constate également des augmentations significatives de production en Argentine (+64 $\%$ en 10 ans). Cependant, le marché potentiel pour le miel de pays africains reste important, selon une étude de l'USAID sur le miel exporté depuis les pays Africains vers l'Union Européenne et les Etats Unis. En effet, le miel de pays Africains reste encore pure et sans additifs, très prisé sur les marchés de niche et par les consommateurs connaisseurs de l'Europe et des Etats Unis (Rongead, 2014). Cette image d'un miel guérisseur persiste malgré quelques 
cas anecdotiques d'allergie ou d'intoxication. Cependant, les étapes d'élaboration du miel sont complexes et susceptibles d'être altérées par les activités humaines, de manière volontaire ou non (Lequet, 2010). Nombreux micro-organismes pathogènes; tels que Eschenchia coli, Staphylococcus aureus, Kiebsiella pneumoniae, Listeria monocytogenes et Campylobacter jejuni, ont été signalés comme agents responsables de maladies d'origine alimentaire et d'altération des aliments (Abbes, 2014). Alors qu'on attribue au miel un très grand nombre de propriétés thérapeutiques (propriétés antiseptiques, antimicrobiennes, antianémiques et antitussives par exemple). Le miel est doué d'un pouvoir bactériostatique important, de par sa haute teneur en sucres (plus de $95 \%$ de la matière sèche), sa faible teneur en eau libre $(0,50$ à $0,62 \%)$ et en humidité (14 à 20\%), son acidité et la présence de substances à activité antibactérienne (peroxyde d'hydrogène libre et inhibine) (Lequet, 2010).

Ainsi, les produits issus du travail de ce petit insecte sont utilisés depuis des millénaires et leurs emplois sont retrouvés dans de très nombreuses civilisations et autres croyances. Formidablement bien organisées en société, ils représentent un sujet d'études forcément intéressant tout en apportant plaisir, santé, bonheur, revenus à tout un monde. De plus en plus pratiquée dans le monde l'api thérapie ou l'usage médical de ces produits de la ruche fait l'objet de plusieurs études scientifiques mais qui restent toutefois encore trop peu nombreuses ou incomplètes (Blanc, 2010).

L'objectif de l'étude présente vise à évaluer la qualité sanitaire des échantillons de miel ainsi que leurs propriétés physico-chimique et sensorielles.

\section{I-Matériels et Méthodes \\ I-1-Matériel biologique}

La présente étude porte sur cinquante échantillons de miel collectés dans la région du Worodougou, Séguéla (Côte d'Ivoire) et dans la ville de Daloa. Les analyses ont été réalisées au laboratoire d'Agrovalorisation de l'Université Jean Lorougnon Guédé, Daloa, Côte d'Ivoire.

\section{I-2-Méthodes}

\section{I-2-1-Méthodes d'échantillonnage \\ I-2-1-1 Sites visités}

Trois sites de production de miel ont été échantillonnés dans la région du Worodougou, Séguéla (Côte d'Ivoire). Le premier point de prélèvement des échantillons était le bassin à miel et le second point les fûts à miel pour les trois localités visités BOBI (MB), FORONA (MF) et WONGUE (MW). Les échantillons de miels MC ont achetés dans deux supermarchés de la ville de Daloa (Sup1 et Sup2) (Tableau I). 


\section{I-2-1-2-Échantillons de miel}

Cinquante (50) échantillons de miel ont été collectés en février et mars 2017. Tous les échantillons ont été collectés de manière aseptique dans des flacons stériles de $100 \mathrm{ml}$ et ont été regroupés en fonction de leur origine. Ces échantillons ont été conservés à température ambiante et ont été traités dans les deux mois suivant la collecte.

\section{I-2-2-Analyses microbiologiques}

La recherche et le dénombrement des germes ont porté sur la flore aérobie mésophile totale, les coliformes totaux, les levures et moisissures, la recherche de $\mathrm{S}$ aureus et des salmonelles.

\section{I-2-2-1-Méthode de préparation des milieux de cultures}

Les milieux de cultures ont été préparés selon les recommandations des fabricants.

\section{I-2-2-2-Préparation des échantillons}

Elle a commencé par la préparation de la solution mère (SM). Elle a consisté d'abord à préparer $225 \mathrm{~mL}$ de l'EPT (eau peptonée tamponnée). Puis $25 \mathrm{ml}$ de chaque échantillon de miel a été ajouté au $225 \mathrm{~mL}$ de l'EPT. Pour la dilution, $1 \mathrm{~mL}$ de la SM est prélevé et ajouté à $9 \mathrm{~mL}$ d'eau distillée stérile afin d'avoir une dilution 10-1. $1 \mathrm{~mL}$ de la dilution 10-1 est prélevé à nouveau et ajouté à $9 \mathrm{~mL}$ d'eau distillée stérile pour obtenir la dilution 10-2.

\section{I-2-2-3-Ensemencement des milieux de culture}

Au cours de l'analyse microbiologique des échantillons de miel, plusieurs milieux de culture ont été ensemencés. Un ensemencement dans la masse a été fait avec les milieux Plate Count Agar (PCA) et violet red bile glucose agar (VRBG), puis un ensemencement en surface a été fait avec les milieux Baird Parker, Sabouraud + chloramphénicol et salmonella-shigella.

\section{-Ensemencement dans la masse}

$1 \mathrm{~mL}$ de chacun des échantillons dilué est transféré de façon aseptique dans les boîtes de Pétri bien indiquées. Par la suite, $15 \mathrm{ml}$ de gélose PCA ou VRBG en surfusion ont été coulés dans la boîte étiquetée et contenant $1 \mathrm{~mL}$ de culture. Le contenu de la boîte de Pétri a été mélangé de façcon homogène par un lent mouvement circulaire. Les boites sont incubées à $30^{\circ} \mathrm{C}$ pendant $72 \mathrm{~h}$ pour le milieu PCA et à $37^{\circ} \mathrm{C}$ pendant $48 \mathrm{~h}$ pour le milieu VRBG.

\section{-Ensemencement en Surface}

L'ensemencement a consisté à transférer $0,1 \mathrm{ml}$ de la dilution approprié de l'échantillon de façon aseptique sur la gélose. A l'aide d'un étaleur, l'échantillon est étalé de façon homogène à la surface de la boîte. Les 
boites sont incubées à $37^{\circ} \mathrm{C}$ pendant $48 \mathrm{~h}$ pour le milieu Baird Parker et à $30^{\circ} \mathrm{C}$ pour le milieu Sabouraud + chloramphénicol.

\section{-Ensemencement du milieu SS pour isolement des salmonelles}

$0,1 \mathrm{~mL}$ de la dilution a été transféré aseptiquement dans les tubes contenant $10 \mathrm{~mL}$ du milieu Rappaport Vassiliadis (RV) correspondante. Les tubes ont été incubés à $37^{\circ} \mathrm{C}$ puis observés après $24 \mathrm{H}$. Ensuite à l'aide d'une anse de platine stérile une quantité du bouillon est prélevé puis ensemencé sous forme de stries sur le milieu SS. Les boites sont incubées à $37^{\circ} \mathrm{C}$ puis observés après $24 \mathrm{H}$.

\section{I-2-2-4-Dénombrement des colonies}

Un comptage du nombre de colonie est effectué $24 \mathrm{~h}$ et/ou $48 \mathrm{~h}$ après incubation afin d'estimer la quantité de bactérie par $\mathrm{ml}$ dans chaque échantillons de miel à partir des milieux de culture. Le nombre de microorganismes présents dans un échantillon de produit donné s'obtient par la formule suivante selon la norme AFNOR (1996).

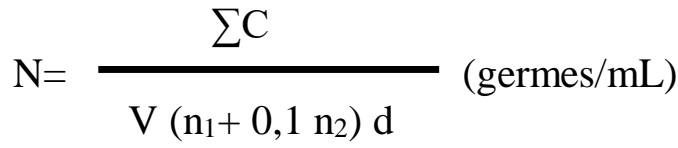

$\mathrm{N}$ : nombres de germes

$\sum \mathrm{C}$ : sommes des colonies

$\mathrm{V}$ : volume de l'inoculum

$\mathrm{n} 1$ : nombres de boites de Pétri comptées à la 1ère dilution

n2 : nombres de boites de Pétri comptées à la 2ème dilution

$\mathrm{d}$ : taux de dilution à la première dilution retenue

I-2-2-5-Normes microbiologiques

Le nombre de microorganisme par $\mathrm{mL}$ ou g a été calculé pour chaque flore étudiée en fonction des échantillons analysés de chaque site, puis comparé à la référence normative des critères microbiologiques.

\section{I-2-3-Analyses physico-chimiques du miel}

\section{I-2-3-1-Détermination de la densité de nos échantillons de miels}

Dix millilitres $(10 \mathrm{~mL})$ de chaque échantillon ont été pesé sur une balance numérique. Après avoir déterminé la masse des $10 \mathrm{~mL}$, la densité du miel a été calculée selon la formule suivante $: \mathrm{d}=\mathrm{m} / \mathrm{v}$.

\section{I-2-3-2-Mesure du potentiel hydrogène d'une solution de miel à l'aide d'un pH mètre}

Dix grammes $(10 \mathrm{~g})$ de miel ont été pesé dans un petit bécher, puis dissous dans $100 \mathrm{~mL}$ d'eau distillée. L'électrode du $\mathrm{pH}$-mètre a été rincée avec de l'eau distillée puis sécher avec du papier joseph. La solution de miel à 
analyser été ensuite placée sous agitation magnétique, avant d'y plonger l'électrode propre et sèche du $\mathrm{pH}$-mètre. A la fin, la valeur du $\mathrm{pH}$ a été lue directement sur l'écran de l'appareil après la stabilisation de cette valeur.

\section{I-2-3-3- Dosage des sucres}

Le dosage des sucres du miel est réalisé par la méthode de Bonnans. A l'aide d'une solution titrée de glucose à $5 \mathrm{~g} / \mathrm{l}$, le poids de glucose nécessaire pour réduire un volume donné de liqueur de Fehling a été déterminé.

\section{I-2-3-4-Determination de l'acidité totale}

Les miels ont été dilués au 1/10 puis leur acidité totale a été dosée par une solution de $0,1 \mathrm{~N}$ de $\mathrm{NaOH}$.

\section{I-2-3-5- Détermination du taux de matière sèche}

Grâce à la méthode de la réfractométrie, le taux de matière sèche soluble a été évalué. La lecture est fait sur l'échelle qui indique la teneur en matière sèche ou «Degré Brix » Pour faire la mesure, le prisme du réfractomètre a été nettoyé et séché, puis le réfractomètre a été réglé à zéro. Le miel à analyser fut homogénéisé et parfaitement rendu liquide, avant de déposé une goutte de ce miel à l'aide d'une spatule, et étalée en couche mince sur la platine de prisme. La lecture a été faite à travers l'oculaire au niveau de la ligne horizontale de partage entre zone claire et zone obscure, et la valeur obtenue pour chaque essai a été notée.

\section{I-2-4-Analyses sensorielles des miels}

Le panel constitutif de cette analyse de couleur a été composé de 74 personnes de tout genre. L'analyse a consisté à observer les différentes couleurs (Incolore, Ivoire, beige, jaune, jaune orangé, marron, marron foncé) entre les échantillons de miel. De ce fait, les échantillons de miel ont été distribués dans différents verres transparents afin de trouver la couleur exacte des miels.

Au cours de cette phase, les dégustateurs ( 74 personnes) ont déterminé également la saveur (acide, sucré, froid, piquant, amer, astringent) des échantillons de miel. La technique a consisté à prendre un peu de miel avec une cuillère, à le mettre dans la bouche en insalivation quelques secondes pour enfin donner la saveur correspondante.

A la suite des autres analyses sensorielles, les dégustateurs ont donné leur appréciation globale des échantillons de miel. La technique consiste à prendre un peu de miel avec une cuillère, à le mettre dans la bouche en insalivation pendant quelques secondes puis dire si le produit est extrêmement désagréable, très désagréable, désagréable, assez désagréable, ni agréable ni désagréable, assez agréable, agréable, très agréable, extrêmement agréable. 


\section{Resultats et Discussion Analyses Microbiologiques}

Cinq (05) germes (FMAT (Flore Mésophile Aérobie Totale), coliformes totaux, levures moisissures, salmonelles, S. aureus) ont été recherchés dans chaque échantillon.

Le miel MB (1-7,17 ufc/ g de miel) (100\%) présente un taux de flore mésophile aérobie totale inferieur à $10 \mathrm{ufc} / \mathrm{g}$ de miel tandis que 100\% MC (39 ufc / g de miel) et 100\% MW (31,5 à 35,31 ufc/g de miel) ont des taux de flore mésophile aérobie totale compris entre $10 \mathrm{ufc} / \mathrm{g}$ de miel et $50 \mathrm{ufc} / \mathrm{g}$ de miel. Le miel MF présente les taux de flore mésophile aérobie totale les plus élevés (56,70 à 66,14 ufc / g de miel) et supérieurs à 50 ufc / g de miel (Tableau II). Les échantillons de miel analysés ne relèvent pas de contamination par les coliformes (Tableau III). Tous les échantillons de miel provenant des sites de production du Worodougou sont dépourvus de levures et moisissures excepté le miel du commerce avec un taux de contamination ( 2 ufc / g de miel) inférieur à $10 \mathrm{ufc} / \mathrm{g}$ de miel (Tableau IV). L'analyse microbiologique montre également une contamination par S. aureus du miel provenant de MW avec un niveau de contamination (3,64 à 4,63 ufc/g de miel) supérieur à $1 \mathrm{ufc} / \mathrm{g}$ de miel (Tableau V). Aucune salmonelle n'a été détectée dans l'ensemble des miels (Tableau VI). Les taux de contamination observés dans la présente étude restent inferieurs à $100 \mathrm{ufc} / \mathrm{g}$ de miel (Figure 1). Par ailleurs, les miels collectés dans les bassins à miel (BM) des différentes localités ont des taux de contamination en germes supérieurs à ceux des fûts à miel (FM).

La source de microorganismes retrouvés dans le miel peut être les pollens, tubes digestifs des abeilles, air, sol et le nectar. Cette contamination peut aussi provenir des traitements subi par le miel aux cours de son extraction et de sa conservation selon plusieurs auteurs CAA (2015), Grabowski et Klein (2015), Kacaniova et al., (2009). Les miels de la présente étude ont enregistré une charge en flore aérobie mésophile variant de 1-5,16 ufc / g de miel pour le miel MB à 56,70 - 66,14 ufc / g de miel pour le miel MW. Ces valeurs sont similaires à celles obtenues par Leticia et al., (2017) sur des miels collectés à plusieurs niveaux du processus d'extraction et de conservation de miel en Argentine (30-54,25 ufc / g de miel). Ces valeurs restent inférieurs à la norme (100 ufc / g). La présence de la flore mésophile est un indicateur que le processus d'altération par les microbes est engagé. Néanmoins, des valeurs faibles n'indiquent pas nécessairement la présence de pathogènes Sutra et al., (1998). Aucun coliforme n'a été détecté dans l'ensemble des miels. Ces résultats sont opposés à ceux de Mouteira et Basso (2014) ayant obtenus 19, $87 \mathrm{ufc} / \mathrm{g}$ de miel pour les coliformes. Ces résultats indiquent que l'extraction des miels a été réalisée dans de bonnes conditions d'hygiènes contrairement à celles de ces auteurs. L'analyse de MC a montré une charge de levures et moisissure de $2 \mathrm{ufc} / \mathrm{g}$ de miel tandis que les autres miels n'ont revelé aucune 
présence de ces germes. Cette valeur est inférieure à celle de Mouteira et Basso (2014) (34,7 ufc / g de miel). Les levures du genre Saccharomyces sont des agents de la fermentation alcoolique qui altèrent les miels. Ces levures proviennent des pollens et des pattes, langues et jabots des abeilles, contaminés au contact des nectaires floraux et éventuellement des fruits mûrs Fléché et al., (2009). Le miel du commerce (MC) présente un risque de fermentation plus accru que celui des autres miels (MB, MF, MW). Cependant, il faut noter que la charge enregistrée pour MC reste largement faible et inférieure à $10 \mathrm{ufc} / \mathrm{g}$ de miel. Seul MW, parmi les miels analysés, a présenté un taux de contamination en S. aureus variant entre 3,67 et 4,63 ufc / $\mathrm{g}$ de miel et supérieur à $1 \mathrm{ufc} / \mathrm{g}$ de miel. Ces résultats sont en accord avec les résultats de Carlos et al. (2017) indiquant que pour S. aureus, les résultats d'aucun échantillon de miel n'est au-dessus de $100 \mathrm{ufc} / \mathrm{g}$ de miel. Leur présence dans le miel $\mathrm{MW}$ peut être due à une mauvaise manipulation. S. aureus est considéré comme une bactérie pathogène majeure, causant des infections. La croissance de $\mathrm{S}$. aureus dans les aliments constitue un risque pour la santé publique parce que certaines souches produisent des entérotoxines dont l'ingestion provoque une toxiinfection alimentaire se traduisant par des vomissements violents accompagnés de diarrhée. Il convient donc de faire des recommandations sur l'hygiène et le contrôle sanitaire aux producteurs de miels (MW) de la localité de Wongué. Aucune salmonelle n'a été détectée dans l'ensemble des miels à l'instar des travaux de Carlos et al. (2017) ayant montré l'absence de salmonelle sp. dans 25g de miel. Ces résultats montrent que les conditions d'hygiène ont été respectées au cours des étapes de conditionnement des différents échantillons de miel. En somme, la qualité microbiologique des miels de cette étude est bonne et similaire à celle de plusieurs auteurs Eskevinho et al., (2012); Velasquez Giraldo et al., (2013), Ndife et al., (2014).

Tableau I: Origine géographique des échantillons de miels

\begin{tabular}{lll}
\hline Code de l'échantillon de miel & Localité de provenance & Chef-lieu de région \\
\hline MB & Bobi & Séguéla \\
MC & Daloa (Commerce) & Daloa \\
MF & Foronan & Séguéla \\
MW & Wongué & Séguéla \\
\hline
\end{tabular}


Tableau II- Dénombrement des Flore Aérobie Mésophile dans les miels

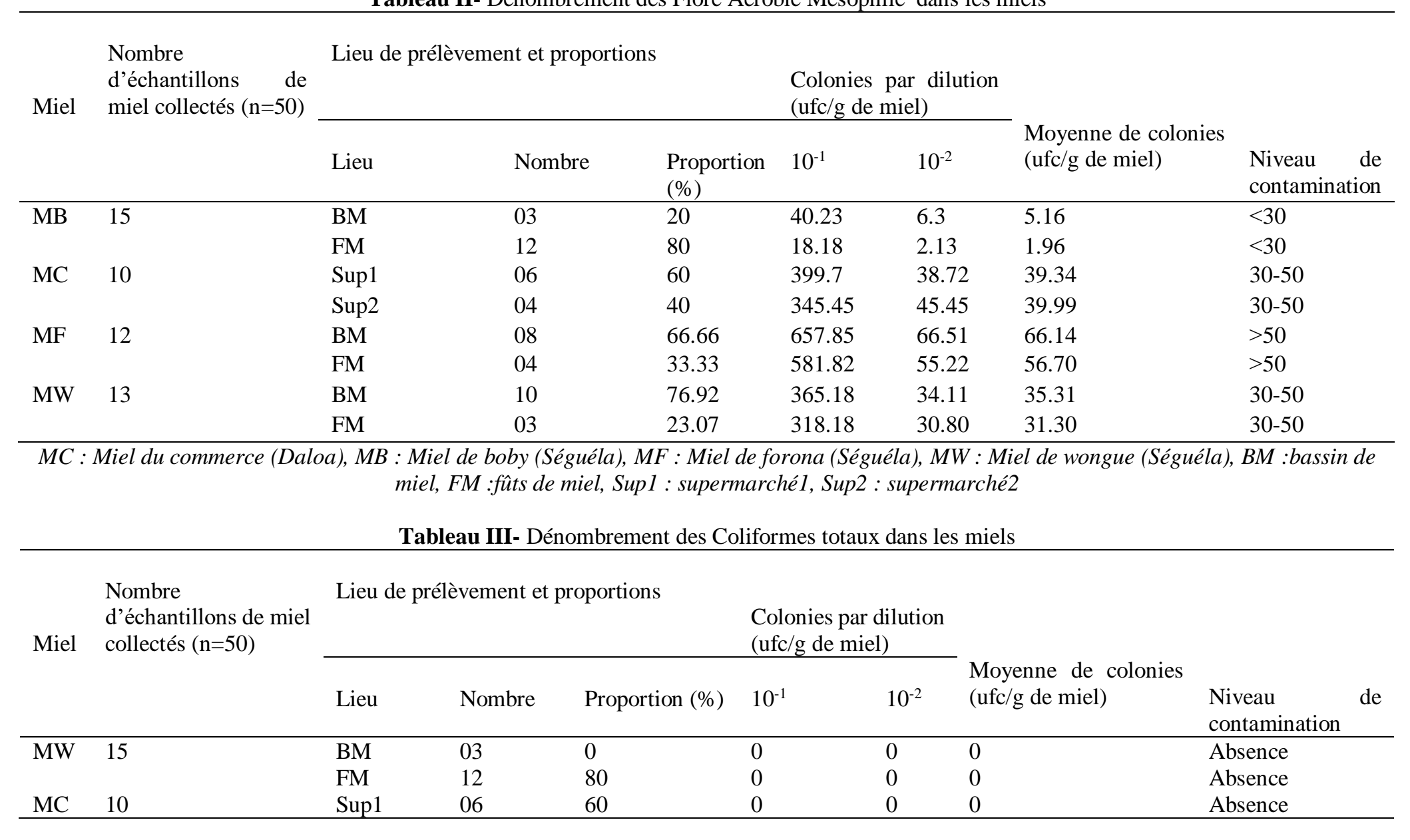




\begin{tabular}{|c|c|c|c|c|c|c|c|c|}
\hline & & Sup2 & 04 & 40 & 0 & 0 & 0 & Absence \\
\hline \multirow[t]{4}{*}{$\mathrm{MF}$} & \multirow[t]{2}{*}{12} & $\mathrm{BM}$ & 08 & 66.66 & 0 & 0 & 0 & Absence \\
\hline & & FM & 04 & 33.33 & 0 & 0 & 0 & Absence \\
\hline & \multirow[t]{2}{*}{13} & $\mathrm{BM}$ & 10 & 76.92 & 0 & 0 & 0 & Absence \\
\hline & & FM & 03 & 23.07 & 0 & 0 & 0 & Absence \\
\hline
\end{tabular}

miel, FM :fûts de miel, Sup1 : supermarché1, Sup2 : supermarché2

Tableau IV- Dénombrement des levures et moisissures dans les miels

Nombre Lieu de prélèvement et proportions

d'échantillons de miel $\quad \begin{gathered}\text { Colonies par dilution (ufc/g } \\ \text { de miel) }\end{gathered}$

Miel collectés $(n=50)$ de miel)

\begin{tabular}{|c|c|c|c|c|c|c|c|c|}
\hline & & & & & & \multirow{2}{*}{$\begin{array}{l}\text { Moyenne } \quad \text { de } \\
\text { colonies (ufc/g } \\
\text { de miel) }\end{array}$} & \multirow[b]{2}{*}{$\begin{array}{l}\text { Niveau de } \\
\text { contamination }\end{array}$} \\
\hline & & Lieu & Nombre & Proportion (\%) & $10^{-1}$ & $10^{-2}$ & & \\
\hline \multirow[t]{2}{*}{ MB } & 15 & $\mathrm{BM}$ & 03 & 20 & 0 & 0 & 0 & Absence \\
\hline & & FM & 12 & 80 & 0 & 0 & 0 & Absence \\
\hline \multirow[t]{2}{*}{$\mathrm{MC}$} & 10 & Sup1 & 06 & 60 & 19.09 & 2.10 & 2.00 & $<10$ \\
\hline & & Sup2 & 04 & 40 & 9.09 & 0 & 0.45 & $<10$ \\
\hline \multirow[t]{2}{*}{$\mathrm{MF}$} & 12 & $\mathrm{BM}$ & 08 & 66.66 & 0 & 0 & 0 & Absence \\
\hline & & FM & 04 & 33.33 & 0 & 0 & 0 & Absence \\
\hline \multirow[t]{2}{*}{ MW } & 13 & $\mathrm{BM}$ & 10 & 76.92 & 0 & 0 & 0 & Absence \\
\hline & & FM & 03 & 23.07 & 0 & 0 & 0 & Absence \\
\hline
\end{tabular}

MC : Miel du commerce (Daloa), MB : Miel de boby (Séguéla), MF : Miel de forona (Séguéla), MW : Miel de wongue (Séguéla), BM :bassin de miel, FM :fûts de miel, Sup1 : supermarché1, Sup2 : supermarché2 
Tableau V- Dénombrement des Staphylocoques dans les miels

\begin{tabular}{|c|c|c|c|c|c|c|c|c|c|}
\hline \multirow[t]{2}{*}{ Miel } & \multirow[t]{2}{*}{$\begin{array}{l}\text { Nombre d'échantillons } \\
\text { de miel collectés } \\
(n=50)\end{array}$} & \multicolumn{3}{|c|}{ Lieu de prélèvement et proportions } & \multicolumn{2}{|c|}{$\begin{array}{l}\text { Colonies par dilution } \\
\text { de miel) }\end{array}$} & \multirow[t]{2}{*}{ (ufc/g } & \multirow[b]{2}{*}{$\begin{array}{lr}\begin{array}{l}\text { Moyenne } \\
\text { colonies } \\
\text { de miel) }\end{array} & \text { de } \\
\end{array}$} & \multirow[b]{2}{*}{$\begin{array}{l}\text { Niveau de } \\
\text { contamination }\end{array}$} \\
\hline & & Lieu & Nombre & Proportion (\%) & $10^{-1}$ & $10^{-2}$ & & & \\
\hline \multirow[t]{2}{*}{ MB } & 15 & $\mathrm{BM}$ & 03 & 20 & 0 & 0 & & 0 & Absence \\
\hline & & FM & 12 & 80 & 0 & 0 & & 0 & Absence \\
\hline \multirow[t]{2}{*}{$\mathrm{MC}$} & 10 & Sup1 & 06 & 60 & 0 & 0 & & 0 & Absence \\
\hline & & Sup2 & 04 & 40 & 0 & 0 & & 0 & Absence \\
\hline \multirow[t]{2}{*}{ MF } & 12 & $\mathrm{BM}$ & 08 & 66.66 & 0 & 0 & & 0 & Absence \\
\hline & & FM & 04 & 33.33 & 0 & 0 & & 0 & Absence \\
\hline \multirow[t]{2}{*}{ MW } & 13 & $\mathrm{BM}$ & 10 & 76.92 & 46.36 & 4.63 & & 4.63 & $>1$ \\
\hline & & $\mathrm{FM}$ & 03 & 23.07 & 36.36 & 3.65 & & 3.60 & $>1$ \\
\hline
\end{tabular}

MC : Miel du commerce (Daloa), MB : Miel de boby (Séguéla), MF : Miel de forona (Séguéla), MW : Miel de wongue (Séguéla), BM :bassin de miel, FM :fûts de miel, Sup1 : supermarché1, Sup2 : supermarché2

Tableau VI- Dénombrement des salmonelles dans les miels

Nombre d'échantillons Lieu de prélèvement et proportions

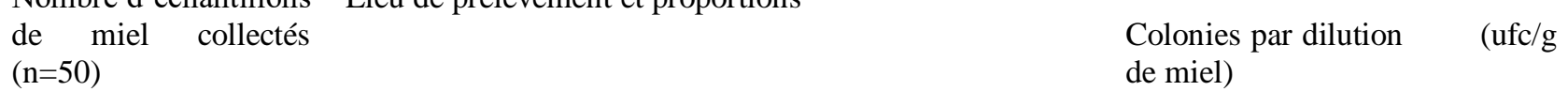

Miel $\quad(n=50)$

\begin{tabular}{lllllll}
\hline Lieu & Nombre & Proportion (\%) & $10^{-1}$ & $10^{-2}$ & $\begin{array}{l}\text { Moyenne de } \\
\text { colonies } \\
\text { de miel) }\end{array}$ & $\begin{array}{c}\text { (ufc/g } \\
\text { Niveau de } \\
\text { contamination }\end{array}$ \\
BM & 03 & 0 & 0 & 0 & 0 & Absence \\
FM & 12 & 80 & 0 & 0 & 0 & Absence \\
Sup1 & 06 & 60 & 0 & 0 & 0 & Absence \\
\hline
\end{tabular}




\begin{tabular}{|c|c|c|c|c|c|c|c|c|}
\hline & & Sup2 & 04 & 40 & 0 & 0 & 0 & Absence \\
\hline \multirow[t]{2}{*}{$\mathrm{MF}$} & 12 & BM & 08 & 66.66 & 0 & 0 & 0 & Absence \\
\hline & & FM & 04 & 33.33 & 0 & 0 & 0 & Absence \\
\hline \multirow[t]{2}{*}{ MW } & 13 & $\mathrm{BM}$ & 10 & 76.92 & 0 & 0 & 0 & Absence \\
\hline & & $\mathrm{FM}$ & 03 & 23.07 & 0 & 0 & 0 & Absence \\
\hline
\end{tabular}

MC : Miel du Commerce (Daloa), MB : Miel de Boby (Séguéla), MF : Miel de Forona (Séguéla), MW : Miel de Wongue (Séguéla), BM : Bassin de miel, FM :Fûts de miel, Sup1 : supermarché1, Sup2 : supermarché2.

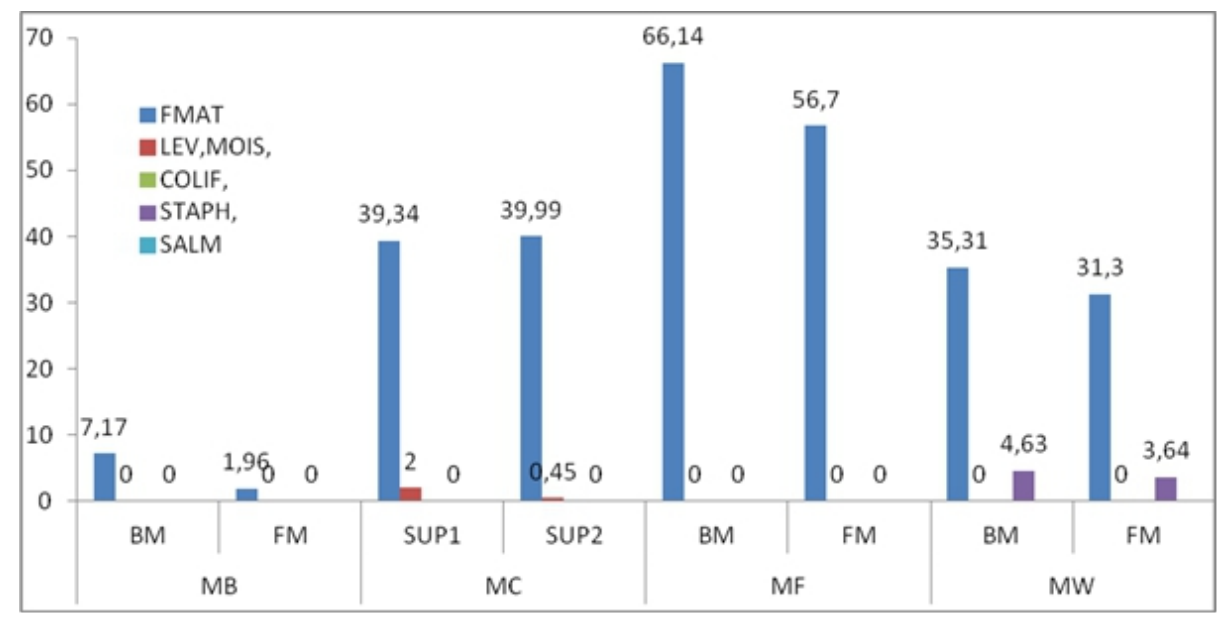

Figure 1 : niveau de contamination des miels 


\section{Composition physico-chimiques des miels}

Les paramètres physico-chimiques évalués sont : la densité, le $\mathrm{pH}$, la teneur en glucose, l'acidité totale et l'extrait sec réfractométrique (ESR) ou degré Brix.

La densité des échantillons de miels varie de 1,06 $\pm 0,069$ à 1,22 \pm $0,089 \mathrm{~g} / \mathrm{cm} 3$. Le MW présente la plus forte densité $(1,22 \pm 0,089 \mathrm{~g} / \mathrm{cm} 3)$, suivi de MF $(1,11 \pm 0,080 \mathrm{~g} / \mathrm{cm} 3)$ et de MB (1,18 \pm 0,024 g/cm3). Le MC (1,06 \pm $0,069 \mathrm{~g} / \mathrm{cm} 3)$ a la plus faible densité. L'analyse statistique ne montre aucune différence significative entre les échantillons de miels $(\mathrm{p}<0,05)$. Les $\mathrm{pH}$ des échantillons varient entre 3,7 $\pm 0,1$ et 4,77 $\pm 0,06$. La plus faible valeur de $\mathrm{pH}$ 3,7 est notée pour $\mathrm{MB}$ et la plus forte pour MW $(4,77)$. L'étude statistique montre une différence significative entre les échantillons $(p<0,05)$. Le MB $(41,71 \pm 6,54 \%)$ a la plus faible teneur en glucose comparé aux autres échantillons (MC, MF, MW). Ces derniers ont des taux de glucose identique $(45,5 \pm 00 \%)$. L’analyse statistique a révélé une différence significative entre $\mathrm{MB}$ et les autres miels $(\mathrm{P}<0,05)$. Les valeurs de l'acidité totale des miels analysés varient de 16,67 $\pm 2,89$ à 33,33 $\pm 2,89 \mathrm{meq} / \mathrm{kg}$. La plus forte valeur d'acidité totale est enregistrée pour MF (33,33 $\pm 2,89 \mathrm{meq} / \mathrm{kg}) ; \mathrm{MW}(33,33$ $\pm 2,89 \mathrm{meq} / \mathrm{kg})$ tandis que la plus faible valeur est pour MC $(16,67 \pm 2,89$ meq $/ \mathrm{kg}$ ). L'analyse statistique montre une différence significative entre MC et les 3 autres échantillons (MB, MF, MW). Les ESR ont varié de $21 \pm 0,44$ à $24 \pm 0,1 \%$ avec une différence significative entre les ESR de MB et ceux des autres (MC, MF, MW). La valeur la plus forte a été enregistrée pour MB (24 $\pm 0,1 \%)$ tandis que les autres échantillons de miels ont présenté des ESR statistiquement identiques (Tableau VII).

Tableau VII: Composition physico-chimiques des miels

\begin{tabular}{|c|c|c|c|c|}
\hline & \multicolumn{4}{|l|}{ Miels } \\
\hline & MB & $\mathrm{MC}$ & MF & MW \\
\hline Densité (g/cm3) & $1,18 \pm 0,024$ (a) & $1,06 \pm 0,069$ (a) & $1,11 \pm 0,080$ (a) & $1,22 \pm 0,089$ (a) \\
\hline $\mathrm{Ph}$ & $3,7 \pm 0,1$ (a) & $4,2 \pm 00(b)$ & $4,4 \pm 00(\mathrm{c})$ & $4,77 \pm 0,06(\mathrm{~d})$ \\
\hline $\begin{array}{l}\text { Acidité totale } \\
\text { (méq } / \mathrm{kg})\end{array}$ & $31,67 \pm 7,64$ (a) & $16,67 \pm 2,89(b)$ & $33,33 \pm 2,89$ (a) & $33,33 \pm 2,89$ (a) \\
\hline $\begin{array}{l}\text { Teneur en Sucre } \\
\text { réducteur }(\%)\end{array}$ & $41,71 \pm 6,54$ (a) & $45,5 \pm 00(a)$ & $45,5 \pm 00(a)$ & $45,5 \pm 00$ (a) \\
\hline ESR & $21 \pm 00(a)$ & $24 \pm 0,44(b)$ & $21 \pm 0,89$ (a) & $22 \pm 0,1$ (a) \\
\hline
\end{tabular}

MC : Miel du commerce (Daloa), MB : Miel de bobi (Séguéla), MF : Miel de forona (Séguéla), MW : Miel de wongue (Séguéla).

\section{Analyses sensorielles des miels}

La mise en évidence des différentes couleurs de miel a été appréciée par un panel de dégustateurs. Il est noté une variation de couleur de miel. Les couleurs ayant attiré plus l'attention des panelistes sont le jaune, le marron et le marron foncée. Le miel du commerce avec sa couleur jaune occupe la 
première place (94\%), ensuite le miel de WONGUE avec une couleur marron foncée $(85 \%)$ et le miel de BOBI avec une couleur marron $(82 \%)$ et enfin le miel de FORONA avec une couleur marron foncée (80\%) (Figures 2a et 2b).

L'observation de sa saveur met en exergue une variation de saveur de miel appréciée par les panelistes. La saveur la plus accentuée est la saveur sucrée et le miel le plus sucré parmi les miels est le miel du commerce (86\%), puis le miel de FORONA (83,1\%), miel de BOBY (74\%) enfin le miel de WONGUE (49\%). A côté, on a la saveur astringente, acide, amère, froide et piquante qui est sensiblement faibles (Figure 3).

La figure 4 présente la variation d'appréciation globale des échantillons de miel. L'appréciation des miels part de très désagréable à extrêmement agréable. Mais les plus appréciées partent d'assez agréable à très agréable. Parmi ces miels, le plus assez agréable est le miel de FORONA (35\%), le plus agréable est le miel du COMMERCE (38\%) et le très agréable est le miel de WONGUE (33\%) (Figure 4).

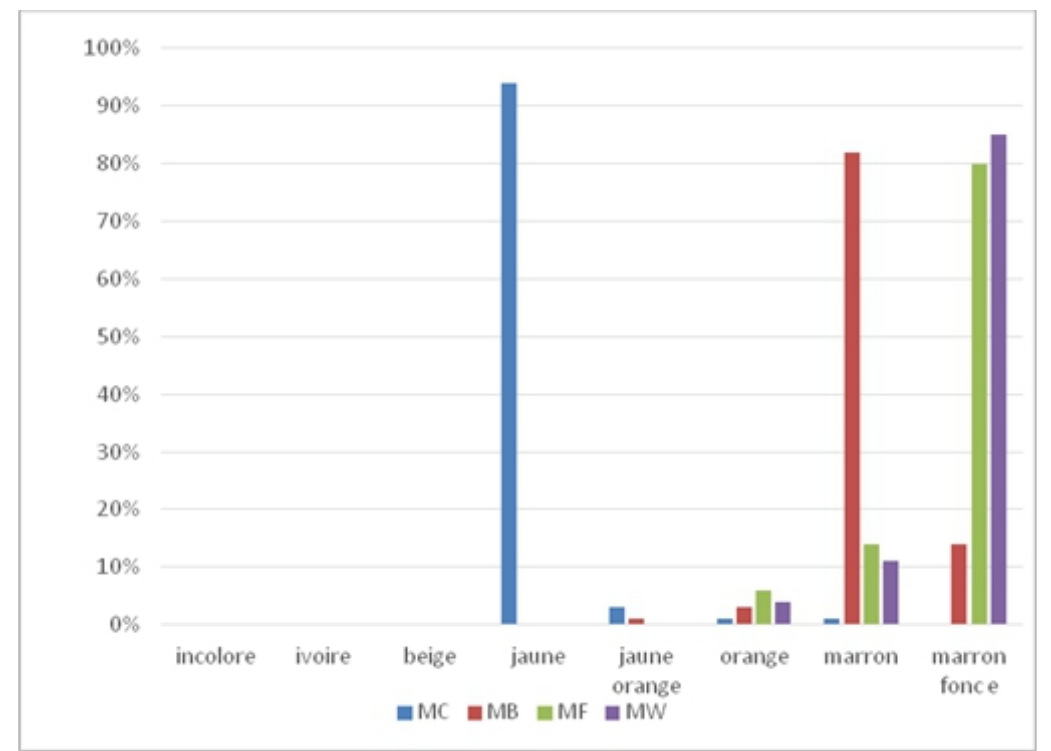

Figure 2a : Couleurs des miels 


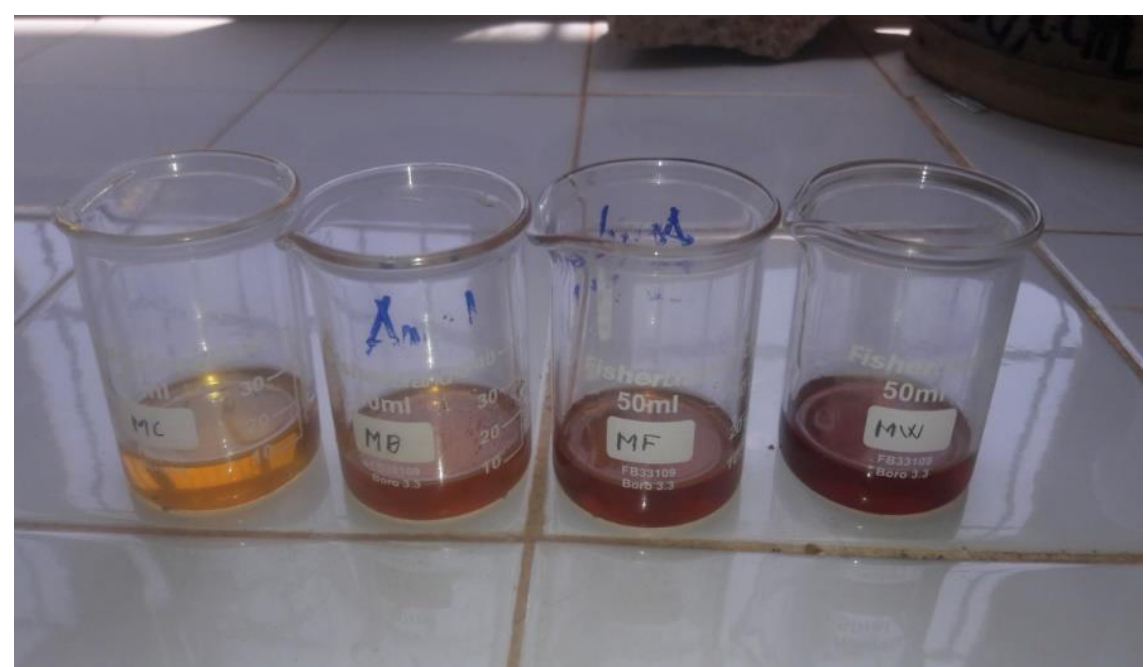

Figure 2b : Couleurs des miels (MC, MB, MF et MW)

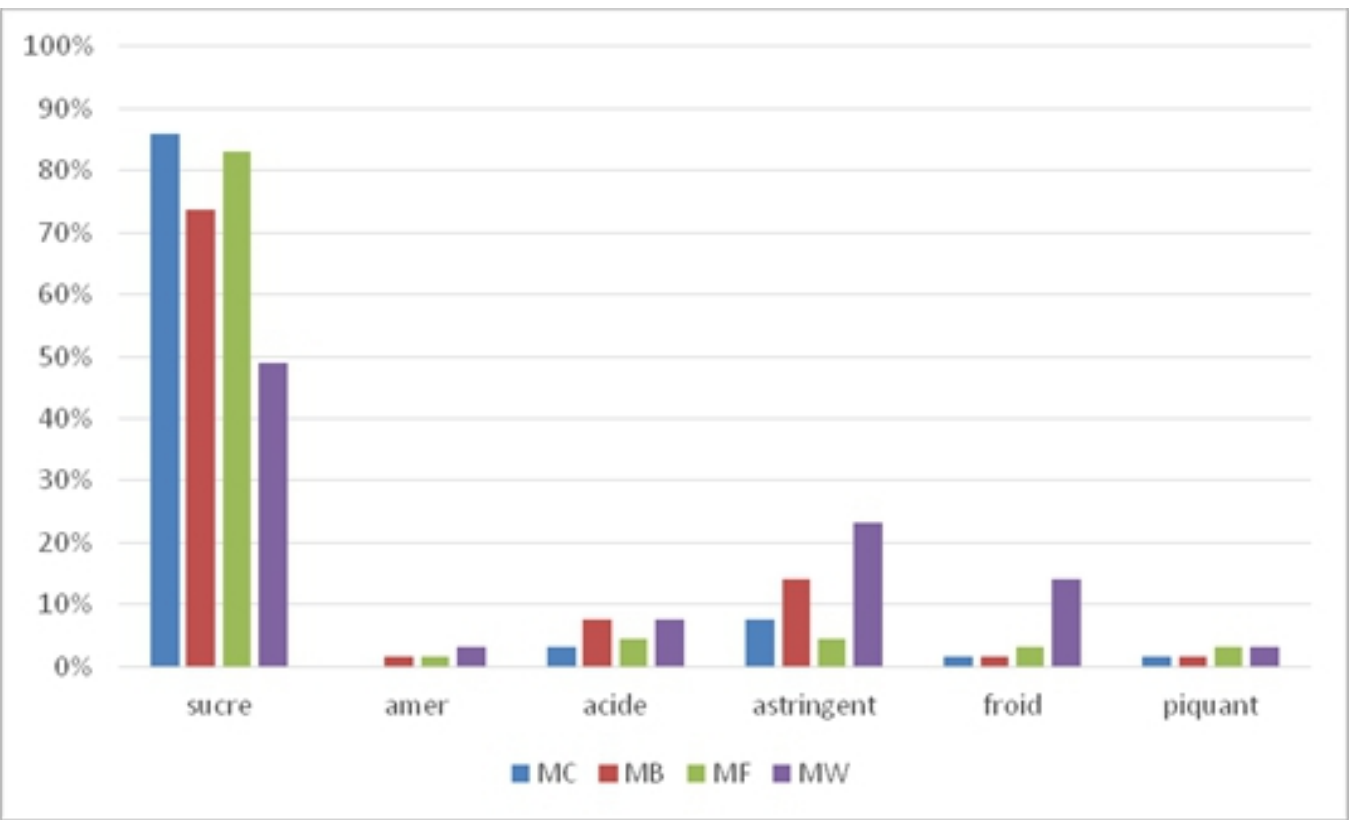

Figure 3 : Saveurs des miels 


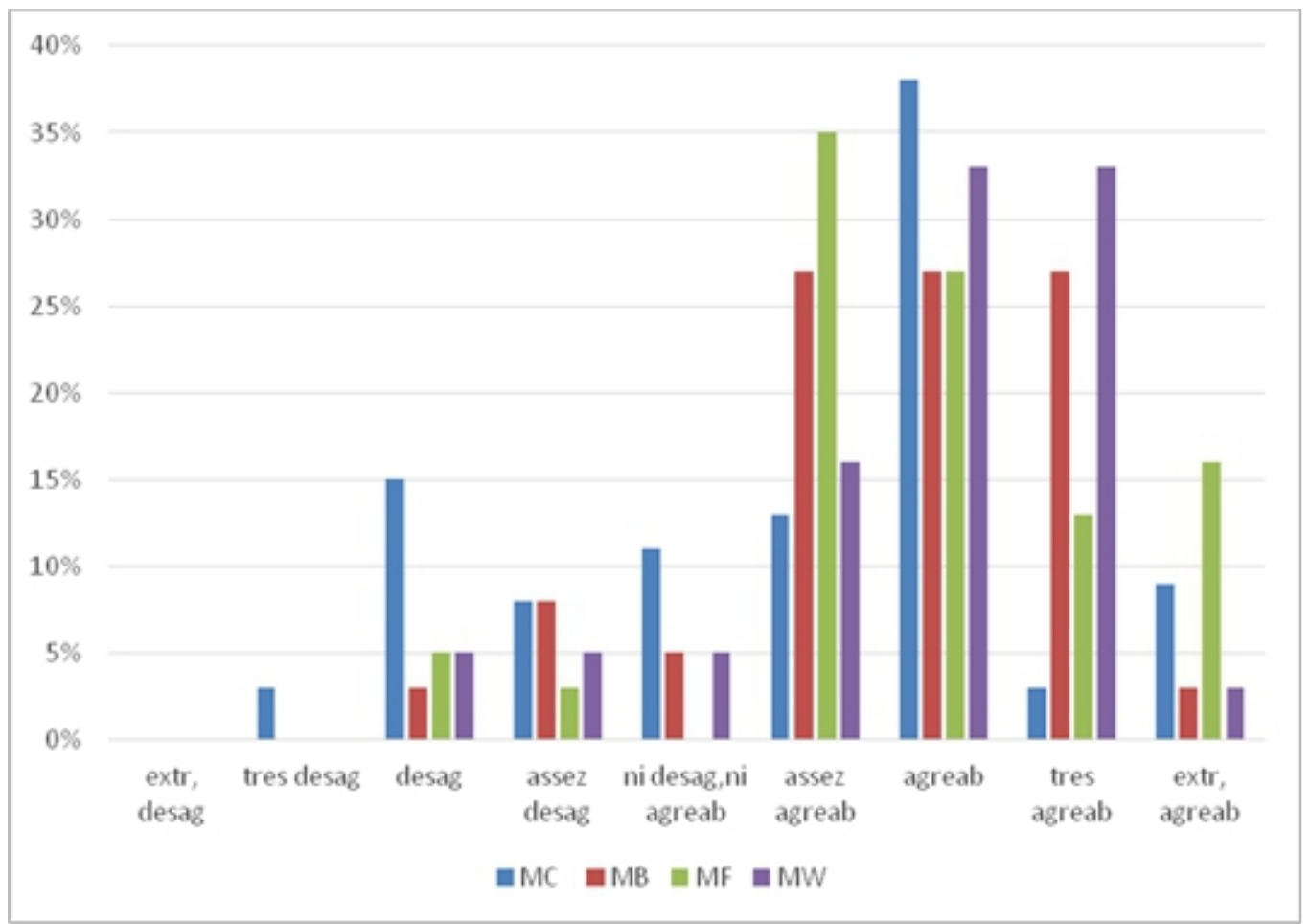

Figure 4: Diagramme d'appréciation globale

La densité des échantillons de miels analysés varie de 1,06 $\pm 0,069$ à $1,22 \pm 0,089 \mathrm{~g} / \mathrm{cm}^{3}$. La plus forte valeur est enregistrée pour l'échantillon de WONGUE avec $1,22 \mathrm{~g} / \mathrm{cm}^{3}$. Cette valeur diffère de celle de Tchoumboue et al., (2007) qui ont trouvé une densité de $1,42 \mathrm{~g} / \mathrm{cm}^{3}$ pour le miel de la zone soudano-guinéenne du Cameroun occidental. Cette différence peut être due à plusieurs facteurs dont la teneur en eau, la température et à moindre degré la composition du miel Gidamis et al., (2004). Les résultats relatifs aux $\mathrm{pH}$ ont donné des valeurs oscillant entre 3,7 et 4,77. Ces pH obtenus sont similaires à ceux trouvés par Alqarni et al., (2012) (3,03-4,73) sur les miels saoudiens; Bath et singh, (1999) sur les miels indiens (3,67-4,48); Ouchemoukh, (2012) $(3,62-4,10)$ sur les miels algériens; ainsi que Silva et al., (2009) $(3,45-4,70)$ sur les miels portugais. Ces résultats suggèrent que le $\mathrm{pH}$ ne dépend pas du milieu géographique. Selon Cavia et al., (2007) le pH du miel est compris entre 3,2 et 5,5, et le $\mathrm{pH}$ des échantillons analysés sont dans la norme (3,74,77). La variation du $\mathrm{pH}$ serait due à la flore butinée, à la sécrétion salivaire de l'abeille et aux processus enzymatiques et fermentatifs pendant la transformation de la matière première Louveaux (1968). Selon Bogdanov, (1995) les miels des fleurs possèdent le plus souvent des valeurs de $\mathrm{pH}$ faibles $(3,3-4,6)$ et les miels de miellats ont des $\mathrm{pH}$ plus élevés $(4,2-5,5)$. On pourrait en déduire que les miels de la présente étude sont des miels des fleurs. Les 
valeurs de l'acidité totale des miels analysés varient de 16,67 à 33,33 meq $/ \mathrm{kg}$. Ces résultats se rapprochent de ceux obtenus par Belhaji et al., (2015) (19,49 à 33,42 méq $/ \mathrm{kg}$ ) sur les miels marocains. Ces valeurs sont aussi proches de celles trouvées par Doukani et al., (2014): 19,56 à 38,91 méq /kg et par Achour et al., (2014): 10 à $40 \mathrm{meq} / \mathrm{kg}$. Les valeurs d'acidité totale des échantillons sont dans la fourchette normale fixée par le Codex Alimentarius (2001) qui est de $50 \mathrm{meq} / \mathrm{kg}$. Cela indique l'absence de fermentations indésirables. La variation de l'acidité totale dans les différents types de miels peut être due selon Perez-Arquillue et al., (1995) à l'origine florale ou à des variations en raison de la saison de récolte. L'acidité est un critère important de qualité, elle donne des indications très importantes sur l'état du miel (Bogdanov et al., 1995). Les teneurs en glucose des échantillons analysés varient de 41,7 à 45,5 $\%$ et sont statistiquement identiques $(\mathrm{p}<0,05)$. Ces valeurs sont supérieurs à celles rapportées par Belhadj et al., (2015) qui sont de 23,35 à 33,37\% et inférieurs à celles trouvées par Achour et al., (2014) et Doukani et al., (2014), qui ont rapporté un taux de sucres réducteurs variant successivement de 61,4 à $79,9 \%$ et de 64,5 et $65,8 \%$. Ces différences sont liées aux types de fleurs butinées par les abeilles (Louveaux, 1968). Les valeurs des extraits secs réfractométriques (ESR) obtenues de cette étude sont proches de celles de Diomandé et al., (2014) (15,6 - 25,13\%) ayant travaillé sur les miels vendus à Daloa. Le MB ayant l'ESR le plus élevé, possède le plus faible taux d'humidité. Cet échantillon serait moins exposé à la fermentation et se conserverait mieux que les autres (Dailly, 2008).

Les couleurs ayant plus attiré l'attention du jury sont le jaune pour le miel du commerce (MC), le marron pour le miel de BOBI (MB) et FORONA (MF) et le marron foncé pour le miel de WONGUE (MC). Lequet (2010) a observé des couleurs de miels variant du beige au noir en passant par le jaune l'orange et le marron tandis que Diomandé et al. (2014) ont vu des miels de couleur noire, jaune et marron au cours de leur travaux respectifs. Ces variations de couleur du miel sont dues à l'origine florale, l'état de cristallisation ou non du miel, la composition chimique du miel et les conditions d'éclairage, la température d'exposition du miel (Lequet (2010); Diomandé et al., (2014). Aussi, de nombreuses études ont montré la corrélation entre l'activité antioxydante du miel et l'intensité de la couleur. Ainsi une couleur foncée indique une forte activité antioxydante et la présence des pigments comme les caroténoïdes et flavonoïdes Beretta et al, (2005). Les quatre miels ont présenté globalement la saveur sucrée. Diomandé et al. (2014) ont noté au cours de leur étude des saveurs amères, astringents et sucré pour de la ville de Daloa. Cette variation de goût des miels dépend de leur composition chimique. La saveur sucrée prononcée du miel dépend de sa haute composition en sucre Delphine (2010). En effet, les glucides ou sucres, sont présents en grande quantité (78 à $80 \%$ ) dans les miels dont la majorité est des 
sucres simples (environ $90 \%$ des sucres totaux) avec une prédominance pour le fructose, davantage que le glucose. A l'état frais, le miel contient plusieurs composés aromatiques qui lui donnent un goût typique. Ces composés étant très volatiles, un miel soumis à un mauvais traitement subira une altération du goût Jocelyn et al., (2013).

\section{Conclusion}

Ce travail a permis d'évaluer la qualité microbiologique et les caractéristiques physico-chimiques et sensorielles de quelques variétés de miel de Séguéla et de Daloa (Côte d'Ivoire). Un taux de flore mésophile aérobie totale de 1-7,17 ufc/ g de miel à 56,70 - 66,14 ufc / g de miel. Les miels analysés ne relèvent pas de contamination par les coliformes. Tous les miels sont dépourvus de levures et moisissures excepté le miel du commerce avec un taux de contamination de $2 \mathrm{ufc} / \mathrm{g}$ de miel. On note une contamination par S.aureus qu'avec le miel MW (3,64 à 4,63 ufc/g de miel). Aucune salmonelle n'a été révélée dans l'ensemble des miels. Par ailleurs, les miels collectés dans les bassins à miel (BM) des différentes localités ont des taux de contamination en germes supérieurs à ceux des fûts à miel (FM).

L'analyse physico-chimique a montré des densités de miels variant de $1,06 \pm 0,069$ à $1,22 \pm 0,089 \mathrm{~g} / \mathrm{cm}^{3}$. Les $\mathrm{pH}$ des échantillons varient entre 3,7 $\pm 0,1$ et $4,77 \pm 0,06$. Le MB $(41,71 \pm 6,54 \%)$ a la plus faible teneur en glucose comparé aux autres échantillons (MC, MF, MW). Les valeurs de l'acidité totale des miels oscillent entre $16,67 \pm 2,89$ et $33,33 \pm 2,89 \mathrm{meq} / \mathrm{kg}$. Les ERS ont varié de $21 \pm 0,44$ à $24 \pm 0,1 \%$.

Les couleurs des miels sont le jaune (MC), le marron (MB) et le marron foncée (MF, MW). La saveur des miels est la saveur sucrée tandis que de façon générale, l'appréciation des miels part de très désagréable à extrêmement agréable. Comparés aux normes internationales, l'on peut affirmer que les miels vendus sur les marchés de Daloa et à Séguéla (Côte d'Ivoire) sont de qualités acceptables.

La détermination des contaminants chimiques, des micronutriments et des vertus thérapeutiques serait nécessaire pour mieux valoriser les miels et autres produits de la ruche en Côte d'Ivoire.

\section{References:}

1. Lequet L. (2010). Du nectar à un miel de qualité : contrôles analytiques du miel et conseils pratiques a l'intention de l'apiculteur amateur. Thèse $n^{\circ} 085$, Université Claude-Bernard - Lyon I, Ecole nationale vétérinaire de Lyon, France, 195p.

2. Rongead. (2014). Miel. Tchad, 10p

3. Abbes A. (2014). Evaluation de l'activité antioxydante des huiles essentielles d'ammoides verticillata « noukha » de la région de 
tlemcen. Mémoire de Master, Sciences de la Nature et de Vie et des Sciences de la Terre et de l'Univers, Université Abou Bekr Belkaid, Algérie, 69p.

4. Blanc M. (2010). Propriétés et usage médical des produits de la ruche. Thèse de doctorat d'Etat, Faculté de Médecine et de Pharmacie, Université de Limoges, 144p.

5. AFNOR, 1996. Microbiologie des aliments - Dénombrement des coliformes thermotolérants par comptage de colonies obtenues à $44^{\circ} \mathrm{C}$, NF V 08 60, Paris, 20p

6. CAA. (2015). Codigo Alimentino Argentino. www. Alimentos argentinos.gov.ar / contenido /marco /capitulos pdf (29/03/2018)

7. Grabowski NT et Klein G. (2015). Microbiology and food-borne pathogens in honey. Crit. Rev. Food. Sci Nutr. www.dx.doi.org

8. Kacaniova M. Melich M, Knazovicka V, Hascik P, Sudzinova J, Pavlicova S, Cubon J. (2009). The indicator Microorganisme value in relation to primary contamination of honey. Lucrari zootechnie si biotechnologii; 42:159-66

9. Letitia A. Fernandez, Carolina Ghilardi, Betiana Hoffmann, Carlos Busso, Liliana M. Gallez. (2017). Microbiological quality of honey from the pampas region (Argentina) throng hout the extraction process, Rev Argent Microbiol; 49 (1). 55-61

10. Sutra L., Federighi M., Jouve J.L. (1998). Manuel de bactériologie alimentaire. Edition Polytechnica, 9p.

11. Monteira MC , Basso IM. (2014). calidad microbiological de la miel proveniente de una sala de extraction Ubicada el partido de Ranchos. In $37^{\text {ème }}$ congreso de la Asoc. Argentina de produccion animal- RAPA, Resumen, Vol.xy, suppl.I.

12. Fléché C., Clément M.-C., Zeggane S., Faucon J.-P. (2009). Contamination des produits de la ruche et risques pour la santé humaine : situation en France. Unité Abeille, Centre national d'études vétérinaires et alimentaires (CNEVA) Sophia-Antipolis, B.P. 111, 06902 Sophia-Antipolis, France. Rev. Sci. Tech. Off. Int. Epiz., 1997, $16(2), 609-619$

13. Carlos V. S., Ruben M. T., Elsa I. Q. R., Ivan N. B., Carlos R. V. Q. (2018).- Microbiol assessment of honey in Mexico calidad. Rev Argent Microbiol; 50 (1): 75-80

14. Eskevinho LM, Feas X, Seija JA, Vazquez- Tato MP. (2012). organic honey from tras-os-Montes Region (Portugal): chemical, salynological,microbiological and bioactive comporunds characterization. Food chem toxicol; 50: 258-64 
15. Velasquez Giraldo M, Velez Acosta LM, Zaluaga Gallego R. (2013). physiochemical and microbiological characterization of Apris melliferd SP honey from. ing.cienc. 9 (18), 61-74

16. Ndife J, Fatimah.K, Tijjani M. (2014). quality assessment of Nigerian honeys from different floral locations, J Food Nutr sci; 2 :162-7

17. Tchoumboue J., Awah-Ndukum J., Fonteh F. A., Dongock N. D., Pinta J. \& Mvondo Z. A. (2007). Physico-chemical and microbiological characteristics of honey from the sudano guinean zone of West Cameroon. African Journal of Biotechnology, 6 (7): 908-913.

18. Gidamis A. B., Chove B. E., Shayo N. B., Nnko S. A. \& Bangu N.T. (2004).- Quality Evaluation of Honey Harvested From Selected Areas in Tanzania With Special Emphasis on Hydroxymethyl Furfural (HMF) Levels. Plant Foods Hum, Nutr, 59 : 129- 132.

19. Alqarni A. S., Owayss A. A \& Mohamed A. A. (2012). Physicochemical characteristics, total phenols and pigments of national and international honeys in Saudi Arabia. Arabia Journal Chemistry, In press.

20. Bath P. K. \& Singh N. (1999). A comparison between Helianthus annuus and Eucalyptus lanceolatus honey. Food Chem, 67: 389-397.

21. Ouchemoukh S. (2012). Caractérisation physicochimique, profils polliniques, glucidiques et phénoliques et activités antioxydantes de miels Algériens. Thèse de Doctorat de Biologie en Biochimie. Université Abderrahmane Mira, Faculté des Sciences de la nature et de la vie, Béjaia, pp.21-90.

22. Silva L. R., Videira R., Monteiro A. P., Valentao P \& Andrade P.B. (2009). Honey from Luso region (Portugal): Physicochemical characteristics and mineral content. Microchem journal. 93:73-77.

23. M. M. Cavia., Miguel A., Fernández-Muiño., Sara R., Alonso-Torre., José F, Huidobro ., María T.Sancho. (2007). Evolution of acidity of honeys from continental climates: Influence of induced granulation Food Chemistry, 100(4):1728 1733

24. Louveaux J. (1968a). Composition, propriétés et technologie du miel. In: Chauvin R. Traité de biologie de l'abeille. Editions Masson et Cie, Paris, Tome 3, pp 277-324.

25. Louveaux. J. (1968).-Composition, propriétés et technologie du miel. In :CHAUVIN R.

26. Traité de biologie de l'abeille. Editions Masson et Cie, Paris, Tome 3, p 277-324.

27. Bogdanov S., Bieri K., Figar M., Figueiredo V., Iff D., Känzig A., Stöckli H et Zü rche K. (1995). Miel: définition et directives pour l'analyse et l'appréciation. Centre Suisse de Recherches Apicoles.126. 
28. Belhaji O., Oumatoi J. \& Zrirai S. (2015). Étude physico-chimique de quelques types de miels marocains. Rev. Mar. Sci. Agron. Vét, 3 (3):71-75.

29. Doukani K.,Tabak S.,Derrriche A., Hacini Z.(2014).Étude physicochimique et phyto-chimique de quelques types de miels Algériens.Revue Ecologie- Environnement 10:37-49.

30. Achour H. \& Khali M. (2014). Composition physicochimique des miels algériens: Détermination des éléments traces et des éléments potentiellement toxiques. Afrique Sci, 10: 127 - 136.

31. Codex Alimentarius. (2001). Codex stan 12-1981, Rev. 1

32. Pérez-Arquillue C., Conchello P., Ariňo A., Juan T. \& Herrera A. (1995). Physico-chemical attributes and pollen spectrum of some unifloral Spanish honeys. Food Chem. 54:167-172.

33. Diomandé M., Koko A. C., Kouamé K.B. et Iritié B.M. (2014). Caractérisation Sensorielle et physicochimique vendus à Daloa, Côte d'Ivoire, sympodium des sciences alimentaires, Université Félix Houphouët Boigny Abidjan cocody, 13 p.

34. Dailly H. (2008). Cristallisation du miel, le savoir et le faire, revue « Technique ». 3: 24-28.

35. Beretta J., Giangiacomo G., Ferrero M., Orioli M. And maffeifacino R. (2005). Standarization of antioxidantproperties of honey by a combination of spectrophotometric/fluorimetricassays and chemometrics. An. Chimica Acta ; 533 :185-191.

36. Delphine Irlande. (2010). Le miel et ses propriétés Thérapeutiques. Utilisation dans les plaies cutanées, 25p.

37. Jocelyn M., Jean N., Émile H. (2003). Les HMF et la qualité du miel. L'abeille, service de zootechnie, mapaq, 15(2) 4p. 